\title{
WEARABLE MIXED REALITY SOLUTIONS FOR INDUSTRIAL PLANTS AND PRODUCTION LINES
}

\author{
Agostino G. Bruzzone $^{(\mathrm{a})}$, Marina Massei ${ }^{(\mathrm{b})}$, Kirill Sinelshchikov ${ }^{(\mathrm{c})}$, Francesco Longo $^{(\mathrm{d})}$, Matteo Agresta ${ }^{(\mathrm{e})}$, \\ Luciano Di Donato $^{(\mathrm{f})}$, Cesare Di Francesco ${ }^{(\mathrm{g})}$ \\ (a) Simulation Team \\ (b), (c) DIME University of Genoa \\ ${ }^{(d)}$ MSC-LES, University of Calabria \\ ${ }^{(\mathrm{e}),(\mathrm{g})}$ Liophant Simulation \\ ${ }^{(f)}$ INAIL \\ (a), (c), (e), (g) \{agostino.bruzzone, kirill, matteo.agresta, cesare.difrancesco\}@ simulationteam.com \\ (b) massei@itim.unige.it \\ (d) francesco.longo@unical.it \\ (f) 1.didonato@inail.it
}

\begin{abstract}
In the present research project, the authors developed wearable and portable solutions capable to improve safety in production lines by taking advantages of availability of exhaustive and reliable data in modern industrial plants. Indeed, the synergy between Industry 4.0 and cutting edge devices, such as smartphones and headsets for Mixed Reality demonstrated to be potentially used to assist personnel on the shop floor, especially during critical and most dangerous operations. In this paper it is presented an ongoing project devoted to develop such support systems and to evaluate their efficiency in multiple industrial environments.
\end{abstract}

Keywords: Modeling, Simulation, Augmented Reality, Mixed Reality, Virtual Reality, Wearable devices

\section{INTRODUCTION}

Modern industrial plants and production lines are characterized by new generations of control systems that include usually quite advanced equipment solutions including numerous sensors as well as capability to collect and transfer an huge amount of reliable data. Typically. these data allows to better control the situation and to activate innovative service such as production optimization and predictive maintenance. therefore there is a significant need to develop Intelligent Solutions able to filter and elaborate these data in order to extract valuable information. So it is evident that these machines represent a potential value and enable new approaches; these "smart" machines could operate in centralized/decentralized mode, feeding directly other devices and systems with a variety of data. Indeed, these capabilities, often grouped under Industry 4.0 name, are crucial to be competitive in today's market. Obviously, the information obtained from these sources could be used not only to optimize costs and efficiency, but even to improve safety.

\section{NEW DATA SOURCES: IIOT}

Recent IIoT (Industrial Internet of Things) developments are interesting for their capability to extend also already existing capabilities and to gather large amounts of data (Big Data) that enable the introduction of new solutions able to integrate distributed systems and new components within networks. Due to these considerations, it is possible to develop innovative solutions for monitoring, tracking and maintenance in industrial plants and to support operations on them.

The availability of Big Data from production lines and industrial plants, especially regarding machines' state, it is the reason why nowadays it is quite common to test and introduce new solutions based on Artificial Intelligence (AI), Digital Twins, Augmented Reality (AR) and Autonomous multi-domain Vehicles (AxV) (Vignali et al. 2018, Bruzzone et al. 2017, Bruzzone et al. 2016). Indeed, the project proposed in this paper is mostly focused on use of Mixed Reality intended as interactive combination of Virtual and Augmented Reality and it is based on the development of an approach to guarantee reliable, up-to-date and exhaustive information from different data sources.

\section{OBJECTIVES}

As mentioned, the availability of information, generated from rich and reliable data sources, provides numerous opportunities of improvement for different aspects of the plants' activities, from performance and productivity upgrade to enhancement of security and safety. In this particular research the authors address several aspects; indeed, in the proposed case it is expected to improve both safety and work optimization. Information regarding systems' state could be used as warning about potentially dangerous conditions as well as to reduce time of response; furthermore, there are new additional functionalities, such as remote assistance: respect this aspect, for instance, it turns 
possible to provide support to operators in front of the production lines through remote assistance from highly qualified personnel, available at any time in Headquarters; this aspect create a new kind of service able to guarantee highly qualified remote support whatever the distance between remote experts and onsite operators.

To address such aspects, the authors propose portable, mobile and wearable solutions in order to assist operators in performing their daily tasks on the shop floor, along production lines and inside industrial plants. In particular, the idea investigated is related to the development of flexible solutions able to be used over multiple platforms from HoloLens to smartphones and tablets, which might be used not only to display information, but also to acquire and store data about interventions and information related to lesson learned. This research is deriving from multiple industrial initiatives; among them it should be mentioned the WARTEMYS project (Wearable Augmented Reality for Employee safety in Manufacturing sYStems) that aims to analyze how these new tools and solutions impact on safety within industrial plants.

According to these considerations, the following objectives have been identified:

- To define Key Plant Indicators and most important risks faced by operators to be provided within the Mixed Reality (MR) in relation to crucial machines and lines in order to alert users on these aspects

- To develop innovative Software solutions able to integrate Industry 4.0 Equipment with Mixed Reality, Simulation and AI, developing also smart solutions for data acquisition, transfer, elaboration and visualization

- To carry out experiments in order to validate the approach and estimate the potential of alternatove solutions applied on site

\section{AUGMENTED PLANT OPERATOR}

As anticipated, this research focuses on testing innovative solutions to support supervision and maintenance for industrial production line using MR technologies; special attention has been paid to use of Augmented Reality to highlight criticalities and performance during operations. Indeed, one aim of this research was also to evaluate efficiency of various types of wearable and portable hardware and software solutions, considering also limitations imposed by the different kinds of production lines; for instance in some case, these environments could be characterized by excessive temperature, high level of noise, dust or even presence of chemical agents. In particular, the authors are testing solutions that are flexible and portable on different platforms including smartphones, tablets and HoloLens.

Augmented Reality and wearable technologies are very good to alert the shift managers and on-site operators about the necessity of immediate intervention. In this way it is possible to provide intuitive and quick access to the main plant indicators as well as to simplify retrieving of information regarding specific machines and production parameters. However, to create these functionalities it is necessary to integrate portable devices and to let them know their position relative to the line and access common databases. Depending on the particular requirements it would be possible to use Indoor Positioning System (IPS) or by attaching QR "anchors" to predefined places of the plant. IPS is one of the most widespread approaches for positioning inside buildings as well as outside. In fact, it is common to use the satellite positioning in many applications, for example GPS; however, within an industrial plant or along a production line, usually, these signals turn to be weak, there are reflections and bad interferences. Fortunately, today there are various tools that allow to finalize the position using transmitters installed in fixed positions within the plant. For example, IPS based on $\mathrm{Wi}-\mathrm{Fi}$ and Bluetooth signals allow to find the position quite precisely and even with minimum infrastructure investment, otherwise there are specialized and more precise systems that use UWB (Ultra-Wide Band) signals (Yang \& Shao, 2015). In facts, in the proposed case studies, considering they require an average precision level positioning, could use any of the mentioned systems. In some cases, absolute positioning on the production line is not required, hence, to avoid unnecessary investments it is possible to use QR codes. Indeed it should be stated that in specific cases, the QR code scanning could be effectively used as way to confirm that the operator/mobile device has reach a specific point in the plant.

Indeed, in this case, all machines of interest are equipped with such tag, which aids finding exact relative position and rotations of the client device, once the code is found. Furthermore, mobile and wearable devices are typically equipped with Inertial Measurement Units (IMU), which allow to keep control over position even after the loss of tag tracking. Hence, even using tags combined with IMU it is possible, usually, to achieve sufficient positioning capabilities. In any case, the positioning solutions allow to improve efficiency of the operators in many ways and are often a crucial element of a solution involving wearable technologies. First of all, in this way, it turns possible to provide up-to-date information of plant situation to the operators: i.e. alerts, abnormal behavior and machines' state as well as various "symptoms" of possible criticalities, production performance anomalies, increase on defects, etc.. In addition, by transmitting the information about the operator activities to the traditional control room, central office and/or engineering department, it is possible to improve efficiency of the remote supervision to operations. Indeed, the synchronization of wearable technology devices in this process allows to know exactly where an operator is located and even what he is looking at.

This approach could be further improved by creating a Digital Twin of the production line, or of the entire plant, and to move operators in this virtual world. In such case, remote supervisor could have access to all 
plant indicators in a virtual 3D environment and assist the operator on site, having the same vision of the plant (Virtual) as the on-site operator (Real), (see figure 1) (Bruzzone et al., 2019a). Obviously, such synergy of technologies could enable even more benefits.

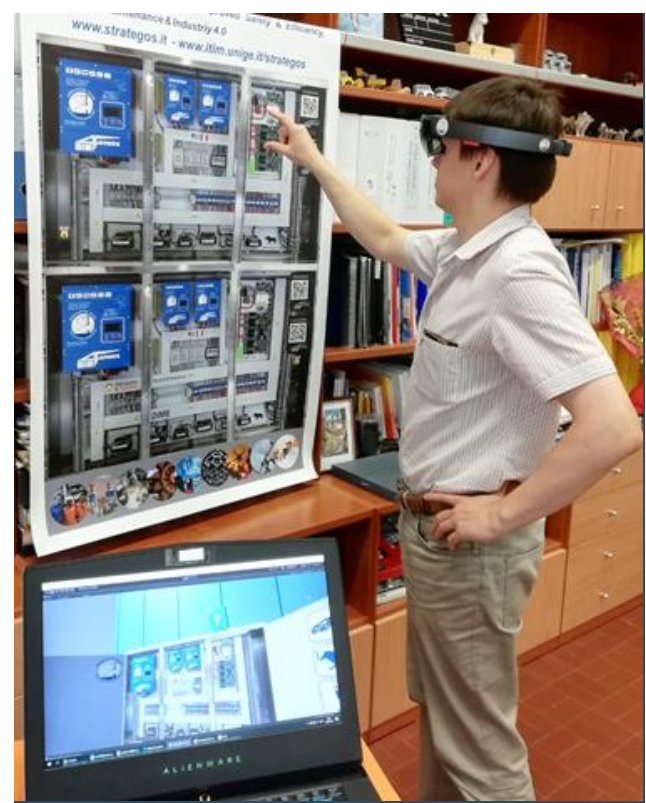

Figure 1: HoloLens for remote supervision, laboratory demonstration

\section{SCENARIOS OF INTEREST}

In order to develop useful and efficient solutions based on this approach, the authors identified several possible cases of application: one related to hollow glass production, while the second is mainly focused on food $\&$ beverages.

\section{Glass Production Line}

Producing hollow glass operates within an "hostile environment"; indeed, the process includes melting the raw material in furnaces at high temperature, hot and cold treatment along noisy production lines, numerous controls and packaging systems. Obviously, the initial parts of such lines have a massive presence of machines and materials at high temperature, while, control and packaging deal with highly fragile and dangerous products, which often causes presence of cracked glass around. Furthermore, automated packaging and transportation systems require to address properly the risk of collisions. In general these environments are considered quite dangerous (BLS, 2017). An interesting peculiarity of this environment is that risks are caused by both machines and products themselves.

\section{Food and Beverage Production Line}

The second case study is related to a bottling line, which covers processes as cleaning and sterilization, bottling and sealing the containers once they are filled up with milk. In this case the main risk factors are related to presence of elevated temperature and pressures in the machines as well as to moving parts.
Quality Control on the Line

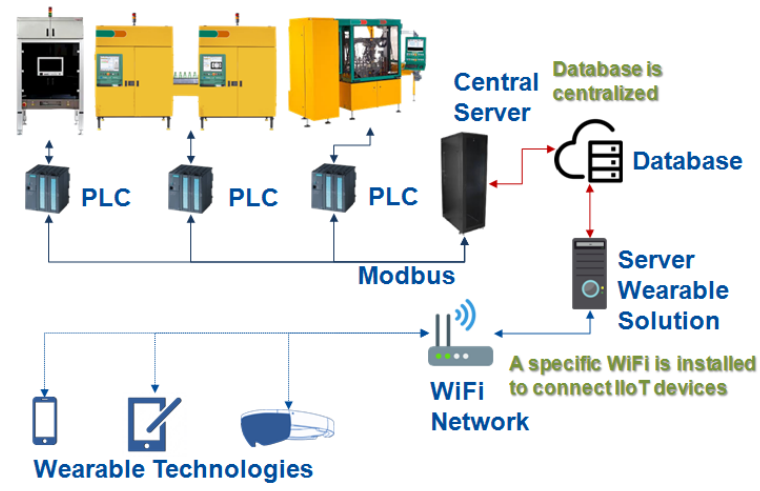

Figure 2: Architecture related of Quality Control along a Production Line

\section{PROPOSED SYSTEM ARCHITECTURE}

To develop flexible solutions for the proposed case studies, it is necessary developed an heterogeneous and robust support architecture integrating different equipment, models and wearable technologies as well as the operators themselves (Longo et al. 2012). Indeed the necessity to create reliable models of the production process it result critical to properly connect them with the virtual representation of the machines and components. An example for online quality control a production plant is summarized in figure 2 . In order to succeed in such task and avoid multiple possible pitfalls, it is essential to define properly the whole architecture that allow this system to perform all required activities, maintaining high level of reliability, efficiency as well as cyber security. For instance, in order to limit access to machinery and keep data acquisition efficient, simple and consistent, it is preferable to have a single access point for such information, for example by providing a web API (Application Programming Interface) or database access. Such centralized approach avoids the redesign the data extraction modules for each different client device, which is very a important aspect considering the variety of access modes and data formats of typical production lines composed by multiple machines produced by different manufacturers. Furthermore, a centralized data source such as a database allows introduction of new functions as well as extension of existing ones, while the entire system could be easily adopted for other production lines and sites. Thus, this approach allows to access the data in a simpler and more effective way, making the project server and clients completely oblivious regarding particularities of access to the different machines and maintaining the highest possible level of security (Tanenbaum \& Van Steen, 2017). Summarizing the proposed solution, the data are extracted and saved in centralized database, periodically retrieved by the server and consequently sent to the clients. In this case the server performs also data elaboration, analyzing the alerts and proposing possible explanations and corrective actions. 


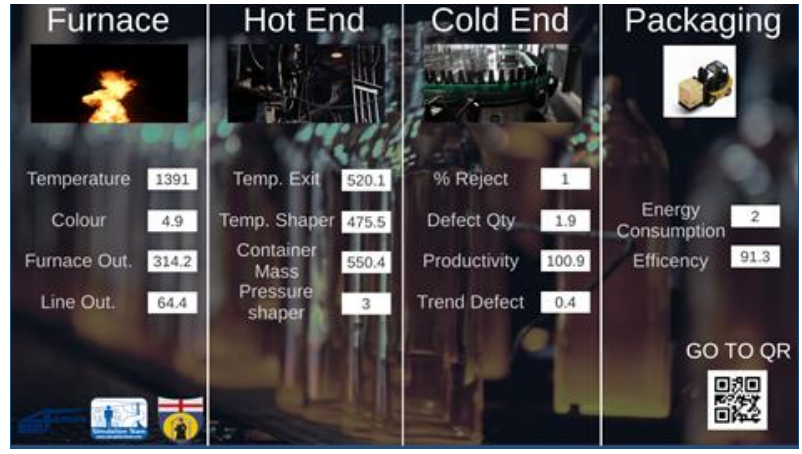

Figure 3: Mobile application displaying main plant indicators

Indeed, the server's functionality could be improved through an Artificial Intelligence module, which would allow, not only to indicate critical situations, but also to foresee them, reducing the risks both for operators and for the plant.

As mentioned, another important aspect of the project is to guarantee security considering that sensible data are shared and operators act inside dangerous environments and within highly cost production processes; in this framework the cyber threats aims not only to security, but also safety issues; for instance, the production line's data are taken directly from the company's servers. Obviously, the maximum degree of security would happen when all the systems were isolated from one another, making however any kind of integration, centralization, control and remote maintenance impossible. Hence, compromises must always be made between safety and efficiency. For these reasons the case study employs only portable solutions capable to maintain high level of data protection. Starting from the proposed architecture it is possible to evaluate the most critical points in terms of data protection and access control.

For instance, considering that in proposed cases, the main server could have access also to some parts of the control system of production lines, it is clear the need to control access to sensitive data. Partially, this issue is addressed by the read-only access to the databases, which reduce drastically possibility of unauthorized modifications to the machines' configuration. In the same time, all but the database's port of the server must be blocked by the firewall. Another criticality related to cyber security is related to the use of wireless networks (Wi-Fi) as support solution for the new system. From this point of view, it is very important to provide high level of data protection and integrity control (Ferguson, 2010). For example, WPA2 protection must be used for connections, while all connected devices must operate within a secure VPN (Virtual Private Network) network (Nguyen, 2018). Obviously, portable devices must be forced to use only the dedicated wireless network. In the scenario of interest such protection level could be sufficient, however, in cases when a public network is required, it could be possible to introduce additional encryption level in order to improve overall protection level, for instance, by introducing probabilistic cryptography layer, such as that one based on ElGamal. Last, but not least: security, but also safety aspects are strongly related to human factors (Bruzzone et al. 2019b). In facts, all security measures were useless if the end user would access external or mobile networks, creating connection bridges between networks, or to install unauthorized applications. Considering this, it is essential implementing access control on the devices, introducing policies and encryption.

\section{USE MODE AND EXPERIMENTATION}

In particular, the authors have identified different possible use mode for these new systems in industrial plants. One is for sure related to support directly the operators within the plant; in this context AI and wearable technologies help the operator to understand quickly problems and criticalities and it is directed over there and guided to solve. Still in this framework the proposed solutions could support remote assistance to operator to solve more complex situation. Another important use mode is related to the idea to adopt these Mixed Reality Solutions to train operators off line and on line and to improve their familiarity with problem solving and interventions on the productions Lines.

From this point of view an example is proposed in relation to the combined use of smartphone and HoloLens to monitor state of the plant; vice versa headset and or CAVE could be also used for remote assistance and training. For instance it is possible to use a tablet to support operators during maintenance, providing them with data on machine alerts and action to be carried out/components to be checked. (see figure 3). Currently, the authors are still testing the new systems and experiencing other functionalities

\section{CONCLUSIONS}

The authors have carried out a research on modern mobile and wearable technologies and their possible integration with Production Plant. The experience was successful both with new industry 4.0 machines and old components inside different kind of production lines and it was possible to identify synergies with available information systems and to integrate data in modern plants in order to improve safety. The project is in active development phase and experimentation are ongoing; however, Subject Matter Experts (SME) have been involved since the beginning and currently they confirm their interest in these solutions, based on preliminary experimental results.

\section{REFERENCES}

BLS (Bureau of Labour Statistics) of United States (2017). Retrieved from www.bls.gov.

Bruzzone, A., Longo, F., Nicoletti, L., Vetrano, M., Bruno, L., Chiurco, A., Fusto, C. \& Vignali, G. (2016). Augmented reality and mobile technologies for maintenance, security and operations in industrial facilities. Proc. of 28th EMSS 2016, Larnaca (Cyprus), September 26-28, pp. 355-360. 
Bruzzone, A. G., Massei, M., Longo, F., Sinelshchikov, K., Di Matteo, R., Cardelli, M. \& Kandunuri, P. K. (2017). Immersive, Interoperable and Intuitive Mixed Reality for Service in Industrial Plants. Proceedings of the 16th International Conference on Modelling \& Applied Simulation, MAS 2017, part of I3M 2017, Barcelona (Spain), September 18 -20, pp. 208-213.

Bruzzone, A.G., Cianci, R., Sciomachen, A., Sinelshchikov, K. \& Agresta, M. (2019a). A digital twin approach to develop a new autonomous system able to operate in high temperature environments within industrial plants. Proceedings of Summer Simulation Conference, Berlin (Germany), July $22-24$.

Bruzzone, A. G., Fancello, G., Daga, M., Leban, B., \& Massei, M. (2019b). Mixed reality for industrial applications: interactions in human-machine system and modelling in immersive virtual environment. International Journal of Simulation and Process Modelling, 14(2), 165-177.

Ferguson, N., Schneier, B. \& Kohno, T. (2010). Cryptography Engineering: Design Principles and Practical Applications. Wiley.

Longo, F., Massei, M., \& Nicoletti, L. (2012). An application of modeling and simulation to support industrial plants design. International Journal of modeling, simulation, and scientific computing, 3(01), 1240001.

Nguyen, H. G. B. (2018). Wireless Network Security: A Guide for Small and Medium Premises, Lathi University of Applied Science

Tanenbaum, A. S., \& Van Steen, M. (2017). Distributed systems. Prentice-Hall, NYC

Vignali, G., Bertolini, M., Bottani, E., Di Donato, L., Ferraro, A. \& Longo, F. (2018). Design and testing of an augmented reality solution to enhance operator safety in the food industry. International Journal of Food Engineering Volume 14, Issue 2, 23 February 2018, Article number 20170122

Yang, C., \& Shao, H. R. (2015). WiFi-based indoor positioning. IEEE Communications Magazine, 53(3), 150-157.

\section{BIOGRAPHIES}

Agostino G. Bruzzone is full professor in Genoa University, President of Simulation Team and currently he serves as Council Chair of STRATEGOS the new MSc in Strategic Engineering and President of the Master Program in Industrial Plant Engineering and Technologies of Genoa University

Marina Massei is active in Modeling and Simulation from over 10 years with crucial roles in R\&D Projects applied to Defense, Homeland Security, Industry and Service to the Society, she has highly qualifications in NATO and Academic Courses related to innovative technologies and acts as Project Controller in R\&D initiatives

Kirill Sinelshchikov has completed the International Master Program in Industrial Plant Engineering and Technologies and he is active as M\&S Researcher working on multiple projects related to industrial applications in Hot Metals, Glass Industry, Ports, Logistics, etc.

Francesco Longo is coordinator of MSC-LES Labs and serve as professor at UNICAL, he is very active in Modeling and Simulation Community since several years with special attention to industrial plant, cultural heritage and logistics.

Matteo Agresta completed his $\mathrm{PhD}$ in Transportation Engineering and works on use of M\&S applied to Logistics, Industrial Plant Engineering and Technologies

Luciano Di Donato is a senior researcher in Italian National Agency for Safety on Work Place with special attention to use of innovative technologies in Industries.

Cesare Di Francesco is active in Liophant conducting researches on innovative solutions based on IoT, Virtual Reality and Simulation applied to Industrial Engineering field 